\title{
A Cognitively Based Approach to Affect Sensing from Text
}

\author{
Mostafa Al Masum Shaikh \\ Dept. of Info. \& Comm. Eng. \\ 7-3-1 Hongo, Bunkyo-ku, Tokyo \\ University of Tokyo, Japan \\ +81-3-5841-6379 \\ mostafa_masum@ieee.org
}

\author{
Prendinger Helmut \\ National Institute of Informatics \\ 2-1-2 Hitotsubashi, Chiyoda-ku \\ Tokyo 101-8430, Japan \\ $+81-3-212-2650$ \\ helmut@nii.ac.jp
}

\author{
Mitsuru Ishizuka \\ Dept. of Info. \& Comm. Eng. \\ 7-3-1 Hongo, Bunkyo-ku, Tokyo \\ University of Tokyo, Japan \\ +81-3-5841-6347 \\ ishizuka@mi.ci.i.u-tokyo.ac.jp
}

\begin{abstract}
Studying the relationship between natural language and affective information as well as assessing the underpinned affective qualities of natural language are becoming crucial for improving the human computer interaction. Different approaches have already been employed to "sense" affective knowledge from text but none of those considered the cognitive structure of individual emotions and appraisal structure of those emotions adopted by emotion sensing programs. It has also been observed that previous attempts for textual affect sensing have categorized texts into a number of emotion groups, e.g. six so-called "basic" emotion proposed by Paul Ekman which we believe insufficient to classify textual emotions. Hence we propose a different approach to sense affective information from texts by applying the cognitive theory of emotions known as OCC model [1] which distinguishes several emotion types that can be identified by assessing the valanced reactions to events, agents or objects described in the texts. In particular we want to create a formal model that can not only "understand" what emotions people wrap with their textual messages, but also can make automatic empathic response with respect to the emotional state detected in the text (e.g. in a chat system). We first briefly describe relevant works and then we explain our proposal with examples. Finally we conclude with future work plans.
\end{abstract}

\section{Categories and Subject Descriptors}

H.5.2 [Information Interfaces and Presentation]: User Interfaces - interaction styles, natural language, theory and methods, prototyping; I.2.7. [Artificial Intelligence]: Natural Language Processing- language models, language generation, language parsing and understanding, text analysis

\section{General Terms}

Algorithms, Human Factors, Languages, Theory

\section{Keywords}

Affective computing, emotions, affective chat, OCC model, affective UI, computational humor

Copyright is held by the author/owner(s).

IUI'06, January 26-February 1, 2006, Sydney, Australia.

ACM 1-59593-287-9/06/0001.

\section{INTRODUCTION AND BACKGROUND}

The words we use reflect who we are and hence the word choice of one's writing serves as a key to one's personality, social situation and affective or attitudinal information conveyed through texts. Furthermore, according to [3] people most naturally interact with their computers in a social and affectively meaningful way, just like with other people. These observations have created an expectation that the future HCI is in themes such as emotions, entertainment, attention, motivation, e-learning etc. So studying the relationship between natural language and affective information as well as assessing the underpinned affective qualities of natural language are becoming crucial for improving interaction with users. Like Liu, et. al. [2] we believe that text is an important modality for sensing affective information because the bulk of computer user interfaces today are textually based. Examples of such applications are the affective text analyzer Empathy Buddy, (see [2] for details), Empathic Chat [4], Information and Tutoring Tools as described in [5], Computational Humor, HAHAcronym, for detail see [6], Affective lexicon, WORDNET-AFFECT as discussed in [7].

For these applications it is necessary to have linguistic resources containing affective knowledge and real-world knowledge of commonsense. In fact all the previous approaches for analyzing texts for affect have commonly employed keyword spotting, lexical affinity, statistical methods, pre-processed models (for storytelling scenario), a dictionary of affective concept and lexicon, or commonsense knowledgebase, but none of those considered the cognitive structure of individual emotions or their appraisal structure. It is also observed that previous attempts, e.g. [2], have categorized texts into a number of emotion groups such as the six so-called "basic" emotion based on "facial expression variables" proposed by Ekman, which we believe are not adequate for classifying emotions expressed by textual information. We also believe that sensing affect from linguistic descriptions or text should consider "phenomenal variables", "behavioral variables" and "cognitive variables" to characterize the structure of emotions usually underpinning text. Phenomenal variables are those variables which could be tracked during the compilation of the text to further associate affective/mental state of the user, for example, time spent to compile a line/paragraph, number of typos per minute etc. could signal about the person's attentiveness etc. In psychology, behavioral variables are some sets of specific behaviors which represent specific emotion. In this case we are particularly interested in "cognitive variables" within the linguistic data. According to a linguistic survey done by Pennebaker [8], across all of the studies described in [8] 4\% were emotional words. This gives us a clue that affective lexicons might not be necessary to express affective states or information by lines of text. Hence 
we might need to employ an emotion model which considers emotions as valanced reaction to consequences of events, actions of agents and different aspects of objects [1] and these phenomena could be detected from linguistic data (e.g. email, chat log, customer feedback etc.) to assess affective information of the user. We believe that the particular emotion a person experiences or describes in text on some occasion is determined by the way he/she construes the world. Thus the attempt of using only commonsense knowledge without the Belief, Desire and Intention (BDI) model of the person/agent may not successfully sense the emotion type and intensity of emotion and the variables that influence the affective sense being conveyed. We also found that the previous attempts to sense affect from text are one-sided in the sense that they do not consider communicating the machine's own emotion with respect to the sensed emotion of the user, although some research has been done to generate affective natural language, for example $[4,5,6]$. For the following sentences, system discussed in [2] can sense the emotions to-be-conveyed in text by using commonsense knowledge and would classify them as "fear", "sad" and "happy" sentences respectively.

(i)Tomorrow I have exam, I am not confident. (ii)I am fine but my younger brother is sick. (iii)I was hoping for good grade and I got it finally.

However, the following set of sentences may not be sensed well, for at least one of the reasons like not considering the cognitive structure of emotions; or not having adequate emotion types for classification; or not considering a BDI model for commonsense because sometimes commonsense is not always "common" to everyone and may vary from person to person, and culture to culture.

(i) I have several assignments pending. (ii)The employee, suspecting he was no longer needed, he might be fired. (iii) Last time I cheated in Pachinko. (iv) I have registered for the conference but I have not booked the flight yet.

Hence the vision of our proposal is twofold: (a) we want to create a computer model that would not only "understand" what emotions people wrap with texts under what circumstances but (b) also automatically generate an empathic reply or response with respect to the emotional state detected (e.g. in a machine chat for e-learning or online counseling; or automatic reply of customer feedback etc.). We would like to incorporate a different approach than in [2], but almost for the same purpose. Particularly we would like to apply the cognitive structure of individual emotions and use the term "emotion type" which distinguishes a set of emotions that can be realized by finding and assessing the valanced reactions to events, agents or objects described in the texts as suggested by OCC Model. Our approach emphasizes cognitive principles underlying the experience of emotions and the characterization of each emotion type.

\section{OUR PROPOSAL}

We are following the OCC Model of emotion. Hence the classes of emotion types we want to identify are listed in [1]. The justification of choosing this model is that it defines emotions as valanced reaction to events, agents or objects and considers valanced reactions as necessary to differentiate between nonemotions and emotions, which seems appropriate for affect sensing from text. Moreover it constitutes goal-, standard- and attitude-oriented emotion appraisal structure. This approach might constitute a new theme to apply natural language processing to the identification of emotion inducing situation, cognitive state of the user, the variables causing emotion etc. and then finally to the categorization of the emotion types concealed in textual descriptions (e.g. in email, story etc.). Formal semantics for different emotion types that we are going to implement for sensing the affect from text are described below. The OCC model defines 22 emotion types specified by a corresponding set of lexical tokens. Due to space limit we are providing the semantics for some of those emotion types, for example,

The sufficient condition for characterizing the "Happy-for" emotion type:

happy-for $(a, x, e, y, t x t) \leftarrow[$ Valanced_Reaction $(t x t) \wedge$

Event_Consequence $(e t x t) \wedge$ Positive_Polarity $(e) \wedge(x<>y$ in $t x t) \wedge$ Desire_For $(e, y)]$

This means, an agent (e.g. emotion sensing program) $a$, senses "happy-for" emotion towards user $x$, for an event/event consequence $e$, found in input text $t x t$, associated with someone else, $y$ if event $e$ is found in the text and a valanced reaction towards that event $e$ is obvious and the event $e$ in the text is having a positive sense in general belief and the event $e$ is associated with someone else, $y$, than the speaker/write, $x$, as well as the event $e$ is desirable for $y$ is true in the text.

Let us explain how this approach is going to work out with examples. Consider the input text "Susan bought a lottery ticket last week, she was very lucky to win the lottery". Using NLP techniques we can get the following, the valanced reaction: "very lucky to win", event consequence: "win lottery" (second statement), polarity of sentence: positive (considering the semantic connotation of object-verb pair gives insight into the associations of the real usage of a word, lottery ticket-bought, and lottery-win), Subject is Susan who is a third person. Considering verbs (buy, win) as events we find "Susan bought" and "Susan wins", and hence events are desirable for the subject. So in this case "happyfor" emotion is senses and agent could generate empathic reply like: "I am really delighted for you, Susan. Are you excited?" etc., that would reflect "happy-for" emotional state of the machine as well as user.

With the input "last time I cheated in Pachinko". The machine may have "pride" emotion type for the consequence of self action of cheating the slot machines provided that agent's own norms allows "cheating in game" praiseworthy and might reply empathically like, "I am really proud of you my friend!!" otherwise if the agent's own norms consider the action as blameworthy, it might reply like "Are you not feeling guilty for doing so?" by classifying the emotion type to "shame or selfreproach". Following we explains "hope" and "fear" emotion.

Hope $(a, \quad x, \quad e, \quad t x t) \leftarrow[$ Valanced_Reaction $(t x t) \wedge$ Event_Consequence $(e, t x t) \wedge$ Desire_For $(e, x) \wedge$ Unconfirmed_Status $(e, t x t) \wedge \quad$ Belief (benefit $a e)]$

For the input, I am wondering if I could buy a new car. The program will sense "hope" emotion by considering "wondering to buy" as a valanced action, "buy a new car" as an event, subject of the sentence is $I$ and subject itself states to buy a car so subject desiring for the event is true. We also find that the status of event is unconfirmed, because of conditionality in the statement (If I could). Let's assume that we have some general beliefs encoded, hence agent's belief "buying a car is beneficial" is true. 
Fear $(a, \quad x, \quad e, \quad t x t) \leftarrow[$ Valanced_Reaction $\quad(t x t) \wedge$ Event_Consequence $(e, t x t) \wedge \neg$ Desire_For $(e, \quad x) \wedge$ Unconfirmed_Status $(e, t x) \wedge \quad\urcorner$ Belief(benefit $a e)]$

For the input, The employee, suspecting he was no longer needed, he might be fired, the program will detect fear by considering "suspecting no longer needed" as valanced reaction, "might be fired" as event consequence. The word "suspecting" implies that the event is not desirable by the subject and the status of the event is unconfirmed by the statement "would be fired". Finally according to agent's encoded belief "loosing a job is not beneficiary" is made true.

We are trying to build a sophisticated sentence parser to find the specific elements required for the emotion semantics to be assessed in terms of cognitive variables and based on these we are trying to classify sentences one of the 22 emotion types. If a sentence fails to satisfy any of the emotion semantics, it will be taken as a non-emotional sentence. We also believe that there are mainly two types of words used in sentences; content and style words. Content words, consisting of nouns, regular verbs, most adjectives and adverbs, focus on the propositional content of an utterance. Style words, consisting of pronouns, prepositions, conjunctions, articles and auxiliary verbs carry the affective meaning and it could be a powerful indicator of people's psychological states. We are considering adjectives and adverb to signify valanced reaction in the text, verb words as the basis of event/event consequence, nouns and pronouns are treated as the agents involved in the sentence to examine different cognitive variables like desirability, prospects, focus etc. of event or object. We are also considering some personality factors for our purpose. Some very interesting findings associated to this can be found in [8] which we also plan to link up while generating empathic reply.

\section{Conclusion}

We are considering sensing the empathic information from the text and/or making empathic reply by programs (e.g. Chatbot) to improve interaction and make text based interface like instant messaging system, yahoo messenger for example, more robust and interactive by associating emoticons or animating own-avatar with respect to sensed emotion to express the affective state. We enlist below a machine conversation (partial) having empathic ingredients. The conversation is made with ALICE [9] Chatbot which is based on AIML (Artificial Intelligence Markup Language) script.

\section{Conversation 1:}

Human: Ok, I am fine now but last night I had a bad dream Computer: Glad to hear it, Human. Why now? Interesting. Really. Do you still have a bad dream?

Human: not really, last night I was falling from a mountain in the dream

Computer: Oh really? Really. Where were you falling from a mountain in the dream? I have been missing you

Human: that was not real but it was really scary though

Computer: Oh Give me an example of real but it was really scary.

Our target is to generate reply something like the following based on sensing the empathy and other personality processes described in [8]

Human: Cool!! But last night I had a bad dream.

Computer: Ahh! Was that scary?
Human: Not really

Computer: Then, why are you afraid of?

Human: I was in a kind of afraid of falling from a mountain!!

Computer: umm, Do you have such bad dream often?

Human: Not that often

In our opinion, machine will be able to generate empathic reply based on our sentence level emotion sensing approach. For the above scenario, "fear-confirmed" emotion type is sensed for the input "Cool!! But last night I had a bad dream". So instead of replying from AIML based pre-scripted answers the machine tries to ask questions about the affective state of the user. As we are planning to implement an online education counselor (text based) for the students to discuss in a chatting manner, we think this sort of affective state awareness and machine reply will improve the interaction and usability of the system. In our system we are going to use ALICE chat-engine and AIML-scripted knowledgebase and apply our emotion sensing approach in between to assess the affective information of the user's input text and ALICE generated reply for that input. If the input text expresses a particular affective state and the output corresponds (for example, if "happyfor" emotion is detected, machine will express "happy-for" emotion, whereas for "anger" machine will express neutral affect), to that state, we accept response give by ALICE otherwise machine makes answer by querying about the affective state of the user. For example, in this case, machine asks, "Ahh! Was that scary?” instead of replying from ALICE- knowledgebase.

\section{REFERENCES}

[1] Ortony, A., Clore, G. L., \& Collins, A., The Cognitive Structure of Emotions, Cambridge University Press, 1988.

[2] Liu, H., Lieberman, H., \& Selker, T., A Model of Textual Affect Sensing using Real-World Knowledge, In Proceedings of the Seventh International Conference on Intelligent User Interfaces, (IUI 2003), Miami

[3] Reeves, B., \& Nass, C., The Media Equation. How People Treat Computers, Television and New Media Like Real People and Places (CSLI Publications, Center for the Study of Language and Information. Cambridge University Press 1998)

[4] Boucouvalas, A. C., \& Zhe, X., Text-to-emotion engine for real time internet communication. In International Symposium on CSNDSP, 2002.

[5] De Rosis, F., \& Grasso, F., Affective Text Generation. In Proc. of International Workshop on Affect in Interactions (IWAI 99), Siena, Italy, 1999.

[6] Stock, O., \& Strapparava, C., Getting Serious about the Development of Computational Humor. In Proceedings of International Joint Conference of Artificial Intelligence (IJCAI-03), Acapulco, Mexico, 2003.

[7] Valitutti, A., Strapparava, C., \& Stock, O., Developing Affective Lexical Resources, Psychology Journal, 2004, Volume 2, Number 1, 61-83

[8] Pennebaker, J.W., Francis, M.E., \& Booth, R.J. (2001). Linguistic Inquiry and Word Count: LIWC 2001. Mahwah, NJ: Erlbaum Publishers

[9] ALICE official web site, http://www.alice.org 